\title{
Exploring the Strain Sensitivity of Image Contrast in Quantitative STEM of $\mathrm{SrTiO}_{3}$
}

\author{
J.Y. Zhang*, J. M. LeBeau**, A. J. D’Alfonso***, L. J. Allen***, and S. Stemmer* \\ * Materials Department, University of California Santa Barbara, Santa Barbara, CA \\ 93106 \\ ** Department of Materials Science and Engineering, North Carolina State University, \\ Raleigh, NC 27606 \\ *** School of Physics, University of Melbourne, Victoria 3010, Australia.
}

High-angle annular dark-field (HAADF) scanning transmission electron microscopy (STEM) is highly sensitive to both the atomic number $(Z)$ and the Debye-Waller factor of the atom columns [1]. Recently, we have demonstrated that excellent quantitative agreement between simulations and experiments can be achieved in HAADF/STEM [2]. This opens the path to examine local changes in Debye-Waller factors, such as in thin films and superlattices, through comparison between experiment and simulation.

In this study, we investigate the strain sensitivity of quantitative HAADF/STEM images. The model systems are strained and unstrained $\mathrm{SrTiO}_{3}$ thin films. Strained $\mathrm{SrTiO}_{3}$ films are grown on (110) $\mathrm{DyScO}_{3}$ substrate surfaces by molecular beam epitaxy. The in-plane tensile lattice strain is 1\%. An epitaxial $\mathrm{SrTiO}_{3}$ film grown on a (001) $\mathrm{SrTiO}_{3}$ substrate served as a reference for unstrained $\mathrm{SrTiO}_{3}$. The biaxial tensile strain affects the $\mathrm{SrTiO}_{3}$ film in two ways: by changing the lattice parameters, which may affect the channeling, and by changing the Debye-Waller factors of the atom columns. For example, previous studies on powders of $\mathrm{BaTiO}_{3}$ indicate an over $50 \%$ increase in the Debye-Waller factor from a $0.5 \%$ lattice strain [3]. Figure 1 shows results from simulations [4] for the column intensities of strained and unstrained $\mathrm{SrTiO}_{3}$, respectively, as a function of thickness. Strained $\mathrm{SrTiO}_{3}$ was modeled using the strained unit cell, both with and without change $(50 \%)$ in the Debye-Waller factor. The results in Fig. 1 indicate that while the change in column intensity due to lattice strain is small, a significant (within the experimental detection limit) difference should be expected for large changes in the Debye-Waller factors.

Simulations were compared quantitatively with experimental HAADF/STEM images, recorded using a FEI Titan 80-300 TEM/STEM field emission microscope with a supertwin lens $(\mathrm{Cs}=1.2 \mathrm{~mm})$ operated at $300 \mathrm{kV}$. The quantitative experiments rely on exact measurements of the local samples thickness, which was obtained from position averaged convergent beam electron diffraction patterns (PACBED). PACBED patterns have been shown to provide precise sample thickness values accurate to within $1 \mathrm{~nm}$ (Fig. 2) and can be recorded in parallel with STEM images without changing the imaging conditions [5].

[1] J. M. LeBeau, S. D. Findlay, X. Wang, A. J. Jacobson, L. J. Allen, S. Stemmer, Phys. Rev. B 79, 214110 (2009). 
[2] J. M. LeBeau, S. D. Findlay, L. J. Allen, S. Stemmer, Phys. Rev. Lett. 100, 206101 (2008).

[3] M. Inagaki et al., J Mater Sci. 8, 312 (1973).

[4] E. J. Kirkland, Advanced Computing in Electron Microscopy, Springer; 2nd ed. edition (August 31, 2010).

[5] J. M. LeBeau, S. D. Findlay, L. J. Allen, S. Stemmer, Ultramicroscopy 110, 118 (2010).

[6] This research was supported by DOE Basic Energy Sciences (DE-FG0202ER45994). We thank Bharat Jalan for the thin film growth.

Fig. 1. Simulated intensity vs. thickness for Sr and Ti columns in unstrained (red), strained (blue), and strained with increased Debye-Waller factor (green) $\mathrm{SrTiO}_{3}$ films.

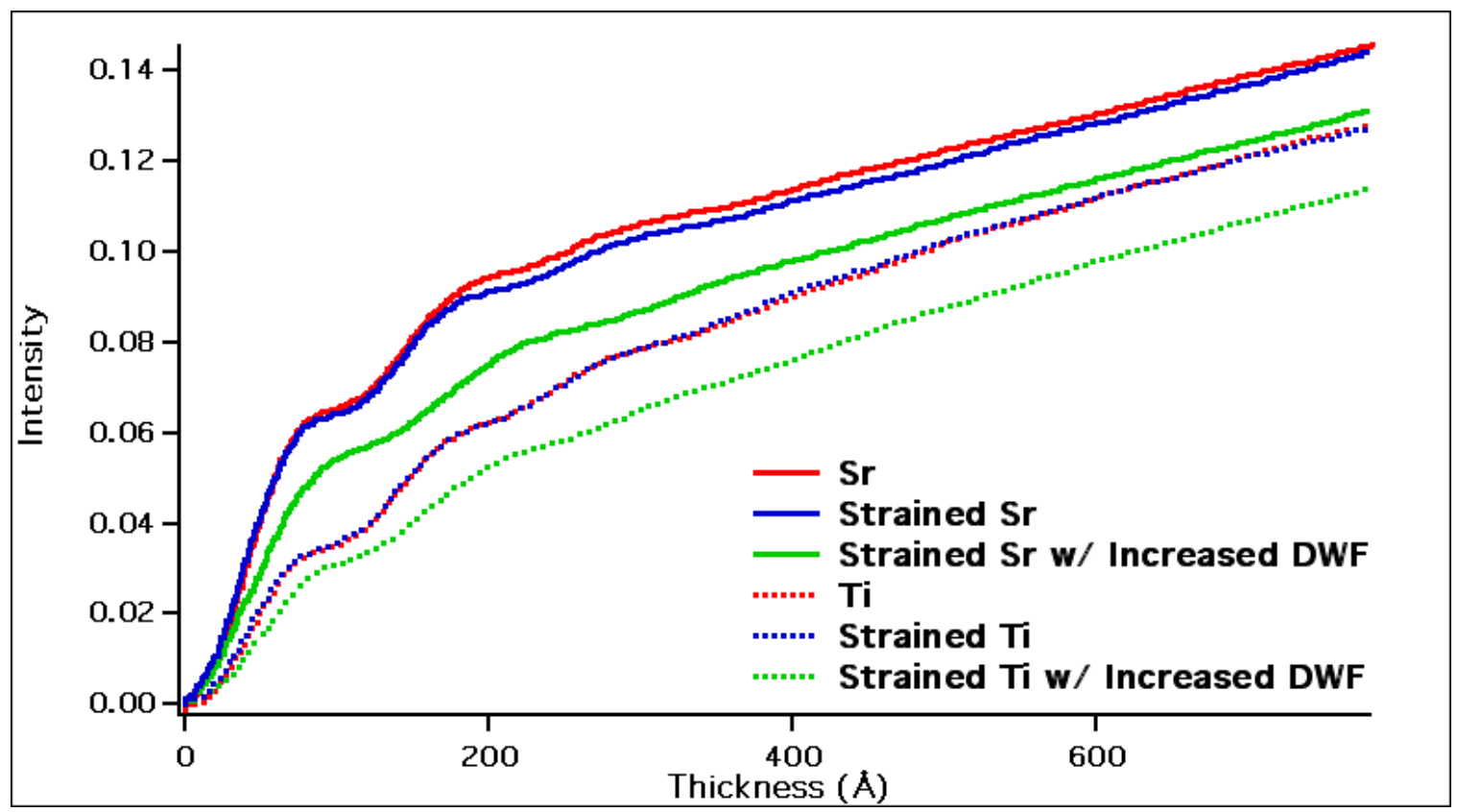

Fig. 2. Simulated PACBED patterns of $\mathrm{SrTiO}_{3}$ for a foil thickness of a) $13 \mathrm{~nm}$, b) $14 \mathrm{~nm}$, and c) $15 \mathrm{~nm}$. In (b) the bottom half has been replaced with experimental image, showing excellent agreement.

(a)

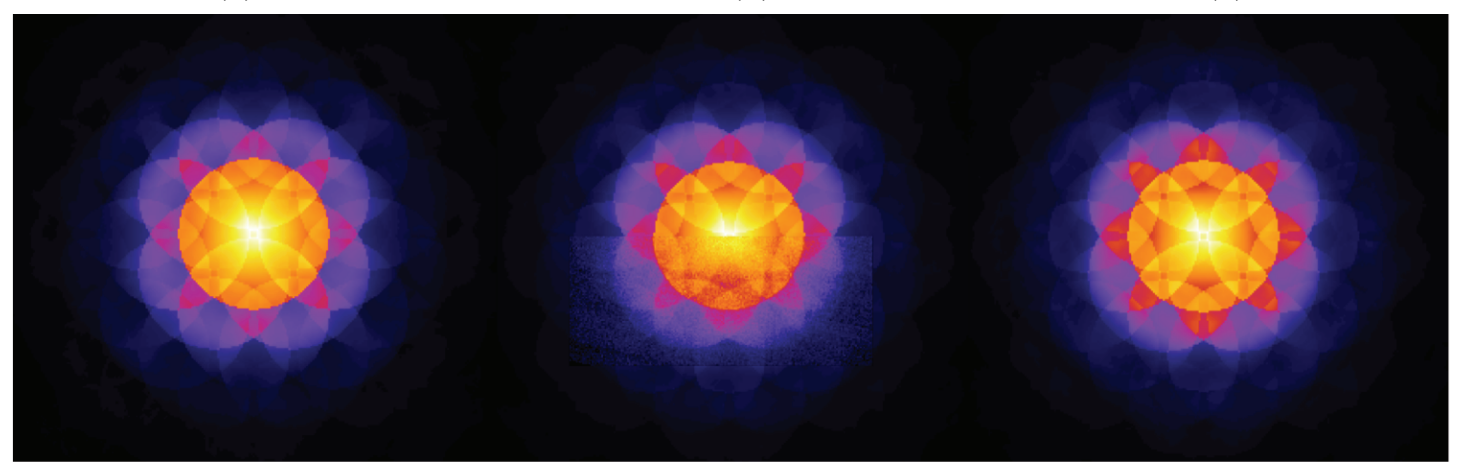

